\title{
STATUS OF SELF-MAGNETIC PINCH DIODE INVESTIGATIONS ON RITS-6 *
}

\author{
K. Hahn, ${ }^{1, \xi}$ N. Bruner, ${ }^{2}$ S. R. Cordova, ${ }^{1}$ I. Crotch, ${ }^{3}$ M. D. Johnston, ${ }^{1}$ J. J. Leckbee, ${ }^{1}$ I. \\ Molina, ${ }^{1}$ B. V. Oliver, ${ }^{1}$ S. Portillo, ${ }^{4}$ J. Threadgold, ${ }^{3}$ T. J. Webb, ${ }^{1}$ D. R. Welch, ${ }^{2}$ and D. Ziska ${ }^{4}$ \\ ${ }^{1}$ Sandia National Laboratories, Albuquerque, NM 87185-1195, USA \\ ${ }^{2}$ Voss Scientific LLC Albuquerque, NM 87108, USA \\ ${ }^{3}$ Atomic Weapons Establishment Reading, Berkshire, RG74PR, UK \\ ${ }^{4}$ KTech Corp. Albuquerque, NM 87123, USA
}

\section{Abstract}

The electron beam-driven self-magnetic pinch diode is presently fielded on the RITS-6 accelerator at Sandia National Laboratories and is a leading candidate for future flash x-ray radiographic sources. The diode is capable of producing sub 3-mm radiation spot sizes and greater than 350 rads measured at $1 \mathrm{~m}$ from the $\mathrm{x}$-ray source. While RITS-6 is capable of delivering up to $11.5 \mathrm{MV}$ using a magnetically insulated transmission line (MITL), the diode typically operates between $6-7 \mathrm{MV}$. Because the radiation dose has a power-law dependence on diode voltage, this limits the dose production on RITS. Coupling this low-impedance $(\sim 40-60 \mathrm{ohms})$ diode to a MITL with similar or higher impedance affects its radiographic potential. The sensitivity in diode operation is compounded by the interaction of evolving plasmas from the cathode and anode, which seem to limit stable diode operation to a narrow regime. To better quantify the diode physics, high-resolution, time-resolved diagnostics have been utilized which include plasma spectroscopy, fast-gated imaging, $\mathrm{x}$-ray $\mathrm{p}-\mathrm{i}-\mathrm{n}$ diodes, $\mathrm{x}-$ ray spot size, and diode and accelerator current measurements. Data from these diagnostics are also used to benchmark particle-in-cell simulations in order to better understand the underlying diode physics. An overview of these experiments and simulations is presented.

\section{I.INTRODUCTION}

The self-magnetic pinch (SMP) diode is a bi-polar relativistic electron beam diode that is presently being characterized as a potential x-ray source for intense, flash radiographic applications. At Sandia National Laboratories (SNL), we are investigating the physics that affects this diode in order to understand and optimize the diode's radiographic potential.
The experiments discussed were performed at SNL utilizing the Radiographic Integrated Test Stand (RITS-6) pulsed-power accelerator shown in Fig. 1 which can provide from 5 to $11.5-\mathrm{MV}$ peak forward-going voltages with 120 to 200-kA peak accelerator currents and 70-ns FWHM electrical pulse widths [1]. On RITS, the SMP diode is capable of producing greater than $350 \mathrm{R} @ 1 \mathrm{~m}$ (rads at $1 \mathrm{~m}$ from the $\mathrm{x}$-ray source) in a sub 3-mm x-ray radiation spot size and a $\sim 50$-ns FWHM x-ray pulse.

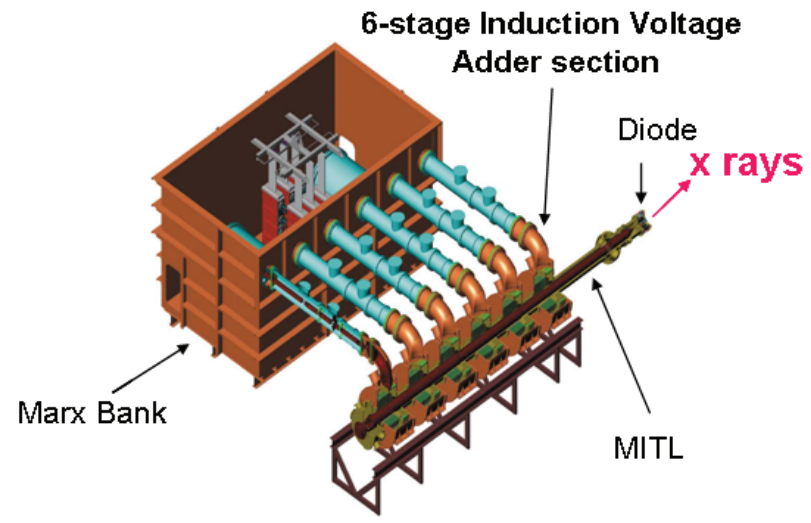

Figure 1. Cut-way view of RITS-6 pulsed-power accelerator.

In this paper, we present an overview of experiments performed on RITS as well as results from numerical simulations and theoretical models. Results presented are for diode operation at 7-MV peak voltage and 150-kA peak current. Experimental data include measurements of the current in the diode region, radiation dose and dose rate, x-ray source spot size, and plasmas in the diode region using gated optical imaging and spectroscopy diagnostics. Simulations using Monte Carlo radiation transport codes and the hybrid particle-in-cell(PIC)/fluid code LSP [2] are also used to compare with data. Diode voltages are inferred from these simulations.

\footnotetext{
* Work supported by Department of Energy's National Nuclear Security Administration under Contract DE-AC04-94AL85000.

६ email: kdhahn@sandia.gov
} 


\section{II.SMP DIODE OPERATION ON RITS-6}

The present understanding of the basic physics of the SMP diode operation is primarily based on a combination of bi-polar relativistic electron beam dynamics; experimental measurements (i.e. currents, $\mathrm{x}$-ray spot size, dose, and dose rates); and advanced numerical simulations.

The typical SMP diode geometry is depicted in Fig. 2. The diode consists of an annular cathode and a large diameter planar anode. The cathode emits an annular electron beam that strikes the anode with electron current densities on the anode surface $\sim 1 \mathrm{MV} / \mathrm{cm}^{2}$. At these current densities, the anode surface is quickly heated $(\sim 5$ ns), forming a dense anode plasma and allowing ion emission. The anode plasma expands into the anodecathode (AK) gap. This expansion leads to a decreasing, if not potentially collapsing, diode impedance. The ions emitted from the anode plasma almost completely neutralize the beam space charge very early in the pulse, but constitute only $\sim 10-20 \%$ of the total current in the diode. Because the beam is not current neutralized, its high self-magnetic field pinches it to a small diameter at the anode. A high- $Z$ material such as tantalum placed at the anode serves as a bremsstrahlung converter to produce $\mathrm{x}$ rays in the forward direction.

Ultimately, the key strategy for the SMP diode is to focus the electron beam to as small a diameter as possible (few $\mathrm{mm}$ ) onto the anode target to produce a small x-ray source size accompanied by high forward-directed radiation dose (100's R@1m) for radiography. It is therefore desirable to understand the diode physics in order to optimize performance.

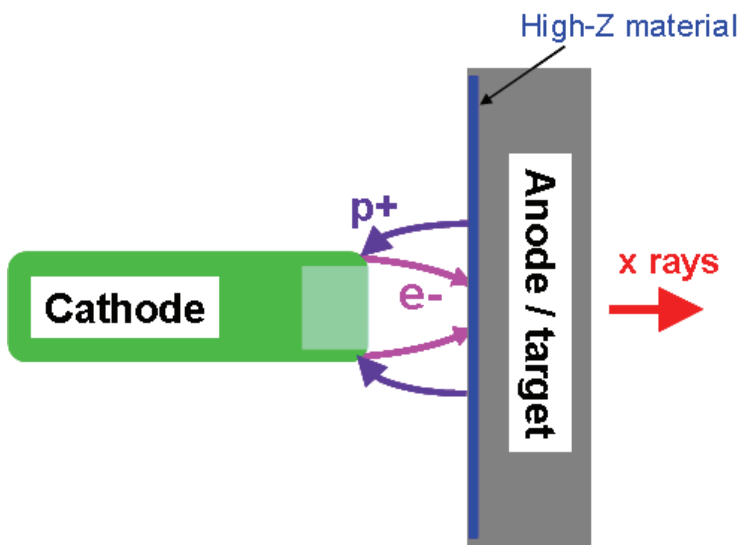

Figure 2. SMP diode geometry.

\section{A. Operating parameters on RITS-6 MITL}

The SMP diode experiments discussed here were all performed using the RITS-6 accelerator [1]. RITS- 6 uses a six-stage induction voltage adder to deliver the power pulse to the diode via a coaxial magnetically insulated transmission line (MITL). In the MITL, the high electric fields $(>100 \mathrm{kV} / \mathrm{cm})$ result in electron emission from the inner (cathode) conductor and the magnetic fields are high enough such that electron orbits bend in upon themselves, remaining near the cathode surface. The current in the MITL is divided between the bound cathode current which flows in the cathode metal conductor and the emitted sheath current layer.

By changing the inner stalk diameter of the MITL and thereby changing the impedance, RITS can be operated in two regimes. In one configuration, the MITL impedance is $\sim 40 \mathrm{ohms}$ and can provide 7.5-MV, 190-kA pulses; and in the other configuration, the MITL impedance is $80 \mathrm{ohms}$ and can provide 11.5-MV, 140-kA pulses. The MITL operating at $40-$ ohms is better matched to the SMP diode impedance which ranges from 40-60 ohms with diode voltages 6-7 MV. When operating with the $80-\mathrm{ohm}$ MITL, the diode is under-matched and consequently also operates between 6-7 MV. The experimental data presented in this paper was obtained using the 40-ohm MITL configuration operating at the 7.5-MV peak MITL voltage.

Because of the presence of sheath current, direct measurements of the voltages in the MITL and diode sections are difficult. However, it is straight-forward to measure the currents throughout the accelerator. PIC simulations using the LSP code are used to compare with the measured currents and infer the diode voltage.

Figure 3 shows an LSP simulation particle plot at $50 \mathrm{~ns}$ (relative to timescale of plot in Fig. 4) of the MITL and SMP diode regions. The MITL is terminated within a large vacuum chamber and the inner cathode conductor is terminated with a large spherical (non-emitting) field shaper and the SMP diode. Macro particles (in blue) represent the electron sheath current launched in the MITL. One of the goals of the field shaper is to limit the sheath flow from interfering with the diode operation, but as shown in the figure, some of the sheath current, 25\% of the total current, wraps around the field shaper. By extending the target upstream of the back vacuum chamber wall, most of the deleterious effects of the sheath current on the diode performance are avoided.

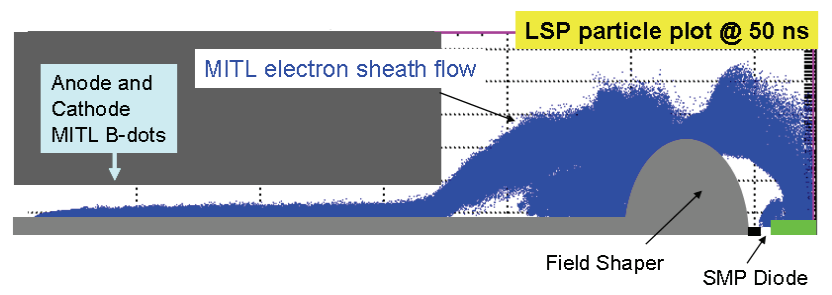

Figure 3. Particle plot from LSP simulation at $50 \mathrm{~ns}$ (middle of pulse) of the MITL and diode sections on RITS-6.

MITL currents are measured using B-dot monitors $~ 2.5$ $\mathrm{m}$ upstream of the diode at the anode and cathode which measure the total anode and bound cathode currents respectively. These measurements are compared to simulations and agree to within $\sim 5 \%$. A MITL voltage can be calculated using the MITL currents with a formula based on the theory of Creedon and Mendel [3-4], which is also compared to the simulated MITL and is in good 
agreement. The peak inferred MITL voltage based on the simulations and calculated voltage using Mendel's formula is $\sim 7.5 \mathrm{MV}$.

In order to properly compare the MITL and diode currents and voltages, the diode impedance must be modeled accurately since this will influence the reflected waves in the MITL that reach the upstream MITL monitors during the pulse. These simulations are the result of an iterative process in which the diode impedance is prescribed in the simulation and then the diode voltage and current are modeled until good agreement is obtained with the measured diode and MITL currents. The simulated and measured diode currents are plotted in Fig. 4. The peak diode voltage is $\sim 7 \mathrm{MV}$, indicating there is some loss $(\sim 0.5 \mathrm{MV})$ in coupling the diode to the MITL.

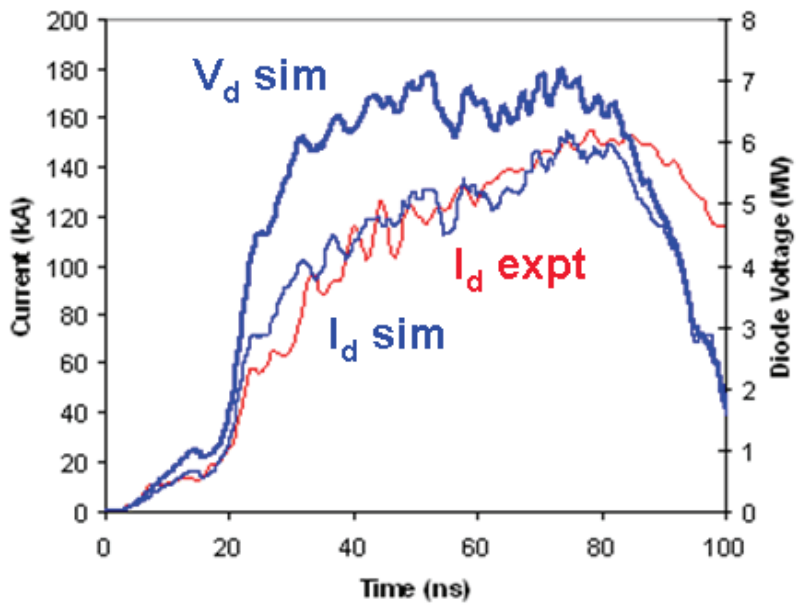

Figure 4. LSP PIC simulation of diode current and voltage compared to measured diode current.

\section{B. Dose Rate Scaling}

Monte Carlo radiation transport codes are used to derive radiographer's equations which provide useful scaling laws to predict the dose based on the current and voltage. Crotch [5] has developed an expression using the MCNP radiation transport code [6] assuming a 40-degree fully-filled cone of electrons incident on an optimal bremmstrahlung target converter which is given by

$$
\frac{\mathrm{D}}{\mathrm{Q}}=-0.06934 \mathrm{~V}_{\mathrm{d}}^{3}+2.3492 \mathrm{~V}_{\mathrm{d}}^{2}-2.952 \mathrm{~V}_{\mathrm{d}}
$$

where D is the dose measured in Rads, Q is the electron charge in $\mathrm{mC}$, and $\mathrm{V}_{\mathrm{d}}$ is the diode voltage in $\mathrm{MV}$.

Another expression proposed by Hinshelwood, et al., [7] based on coupled PIC/Monte Carlo simulations using the integrated tiger series (ITS) code [8] assuming a mono-angular 20-degree electron beam incident on the target is given by

$$
\frac{\mathrm{D}}{\mathrm{Q}}=0.864 \mathrm{~V}_{\mathrm{d}}^{2.2} .
$$

The dose is measured directly in experiments. The electron charge is obtained from the measurements of the diode current which includes both electron and ion contributions. The percentage of total current that is due to electron current is determined from simulations (discussed later) and typically ranges from 80-90\%.

Figure 5 shows a plot of both radiographer's equations for diode voltages up to $10 \mathrm{MV}$. The two expressions convey a similar trend. Over-plotted is data from RITS between 4.5 and $6.5 \mathrm{MV}$. The RITS data agrees well with both expressions. (For this plot, the electron current was assumed to be $85 \%$.)

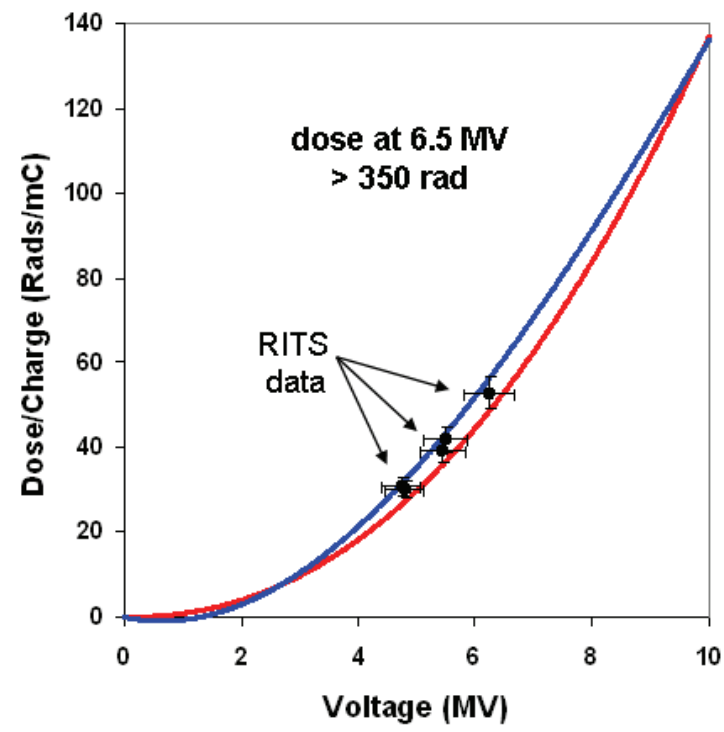

Figure 5. Dose scaling using Radiographer's equations (blue- Eq. (1), red- Eq. (2)) along with RITS data.

Prior to performing experiments on RITS-6, the dose was predicted to be $>350 \mathrm{R} @ 1 \mathrm{~m}$ for a time-averaged diode voltage of $6.5 \mathrm{MV}$, which was measured experimentally. Furthermore, assuming the scaling will remain valid for higher voltages, then >800 R@1m may be achieved at $10 \mathrm{MV}$.

\section{Inference of Voltage and Diode Impedance}

Using the PIC simulations and radiographer's equations, there are two methods for unfolding the diode voltage. The diode voltage inferred from PIC calculations (from Fig. 4) is compared to Eq. 2 and shown in Fig. 6. The peak voltages and pulse-shapes are in good agreement though there are up to +/- 1-MV differences during the pulse. Developing diagnostics for characterizing the diode energy spectrum more directly is an area of research for future diagnostic development.

Using the inferred diode voltages, the diode impedance can be calculated. Figure 7 shows the diode voltage obtained using Eq. (2) (solid black line) and the measured diode current (solid red line) and the calculated impedance (solid blue line) which reveals that the diode impedance falls from $\sim 60$ to $45 \mathrm{ohms}$ during the main 
pulse. The corresponding results from the PIC simulations are plotted in dashed lines. The drop in the diode impedance is indicative of plasma evolution into the AK gap; however, based on the relatively small drop in impedance, they do not seem to significantly affect the diode operation.

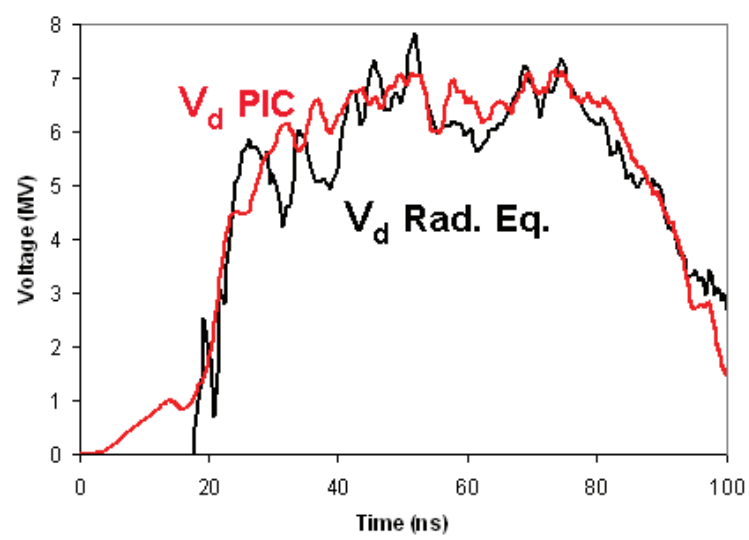

Figure 6. Diode voltage inferred from (black) Eq. (2) and (red) LSP PIC simulation.

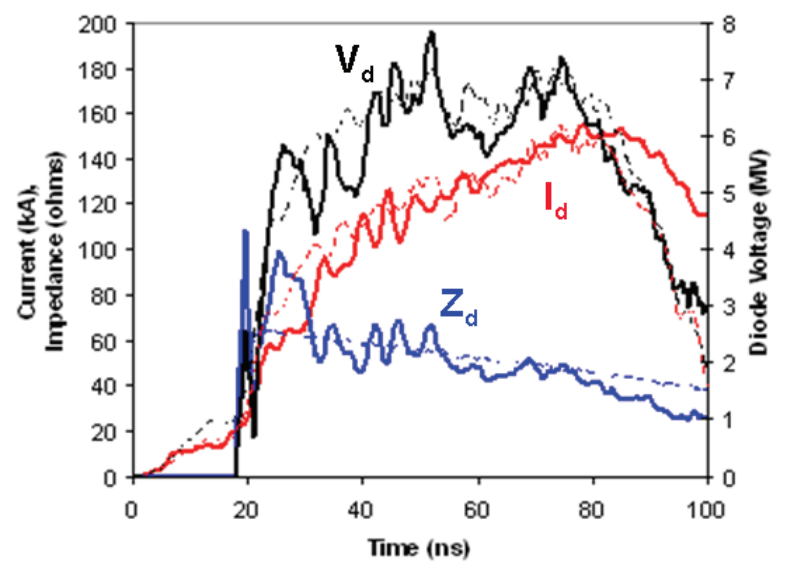

Figure 7. Diode voltage, current and impedance. Diode voltage from Eq. (2) are in solid black line, measured diode current in solid red line, and diode impedance in solid blue line. LSP PIC simulation results are in dashed lines.

\section{III.X-RAY SOURCE CHARACTERISTICS}

Two important quantities for radiography are the x-ray source size and dose. The typical scheme for characterizing the $x$-ray source spot size and dose is shown in Fig. 8. A thick high-Z material rolled edge intercepts the $\mathrm{x}$ rays from the source and projects a shadow onto the planar detectors. Dose and dose rate are measured using thermoluminescent detectors (TLD's) and $\mathrm{p}$-i-n diodes, respectively. The $\mathrm{x}$-ray spot size is measured using an image plate (IP) to obtain the time-integrated source profile as well as a time-resolved spot diagnostic (TRSD) [9]. The top portion of the IP is exposed directly to $\mathrm{x}$ rays and the bottom portion is effectively blocked by the rolled edge. Between these regions is the penumbral shadow region and a line-out taken across this region produces an edge spread function (ESF) profile which is used to characterize the $\mathrm{x}$-ray source distribution. Two rolled edges along the horizontal and vertical axes enable 2-d measurements of the x-ray spot. The temporal spot size is measured using the TRSD which consists of a linear array of scintillator fibers coupled to a streak camera. The TRSD images the penumbral shadow region as function of time in 1-d such that one may obtain an ESF from which the spot can be calculated. The ESF is measured every $\sim 3-5 \mathrm{~ns}$, limited by the fiber response. Because the TRSD is sensitive to dose, it too measures the dose rate though the $\mathrm{p}$-i-n diode has a much faster temporal response $(<1 \mathrm{~ns})$.

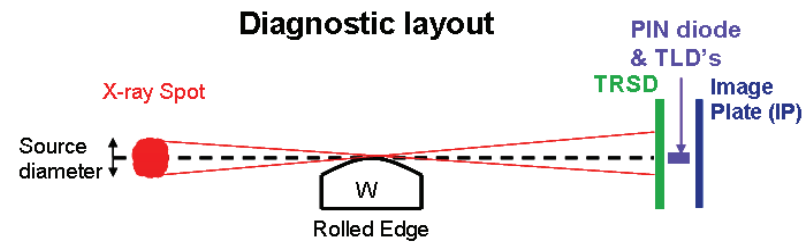

Figure 8. X-ray spot and dose diagnostics layout.

There are several metrics used to quantify the x-ray source spot size. The ESF for a nominal SMP shot is shown in Fig. 9(a). According to the AWE definition [10], the spot size is $2.9 \mathrm{~mm}$ in both the vertical and horizontal directions. Taking the spatial derivative of the ESF yields the line spread function (LSF) shown in Fig. 9(b). A definition of the spot size, based on the LSF is given by $1.4 \mathrm{x}$ the FWHM of the distribution which is $2.2 \mathrm{~mm}$. Based on these definitions, the ESF and LSF spot sizes are nearly equivalent $(\sim 5 \%)$, when the source distribution is Gaussian. Because the LSF spot is $~ 25 \%$ smaller, it indicates that the source is more peaked in the central core. This implies that the source has a potentially higher resolving power for radiography than would be inferred from the ESF spot size definition.

Time-resolved measurements of the average position of the spot in 1-d indicate that the beam is stable on the target during the pulse. As shown in Fig. 10, as the dose rate measured using the TRSD increases, the spot size decreases below $3 \mathrm{~mm}$ and remains below $3 \mathrm{~mm}$ for the duration of the pulse. The spot size minimum of $\sim 2.5 \mathrm{~mm}$ is established during the rising portion of the pulse and increases by $\sim 0.5 \mathrm{~mm}$ during the main pulse. The relative movement of the centroid of the x-ray source is also measured and indicates that movement is typically $<0.25$ $\mathrm{mm}$ which is below the detector resolution. The timeintegrated spot sizes from both the TRSD and IP data typically agree to within $<5 \%$.

The dose rate measured by the $\mathrm{x}$-ray $\mathrm{p}$-i-n diode is shown in Fig. 11. It peaks at $\sim 8$ Rads/ns with a FWHM of $\sim 45 \mathrm{~ns}$. The overall shape is similar to the TRSD dose rate shown in Fig. 10. The integral of the dose rate which 
is calibrated from the TLD measurements yields the dose which is just over350 R@1m.

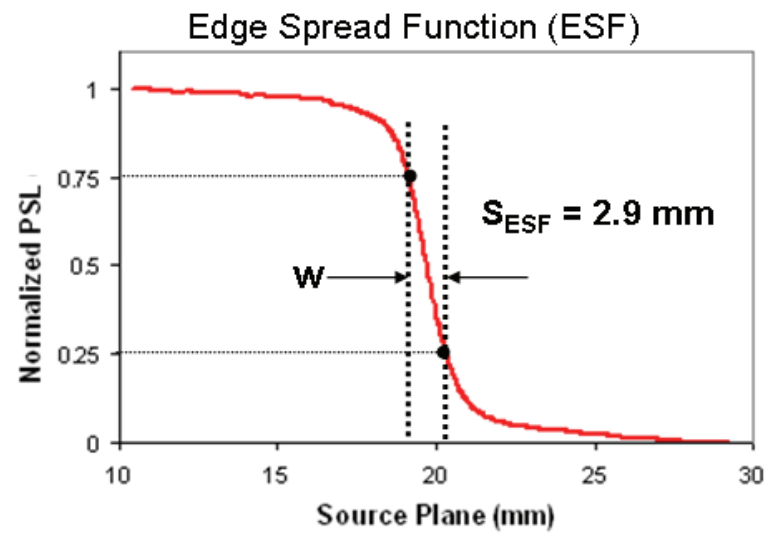

(a)

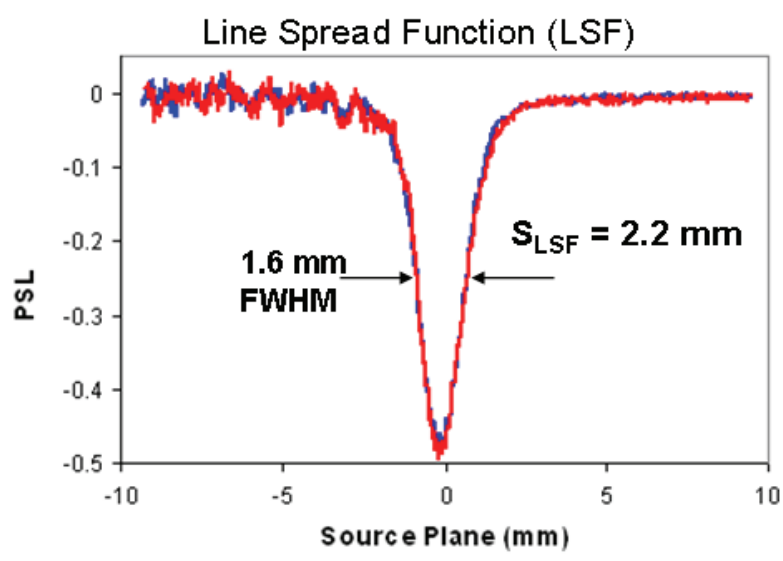

(b)

Figure 9. (a) ESF spot size and (b) LSF spot size measurements.

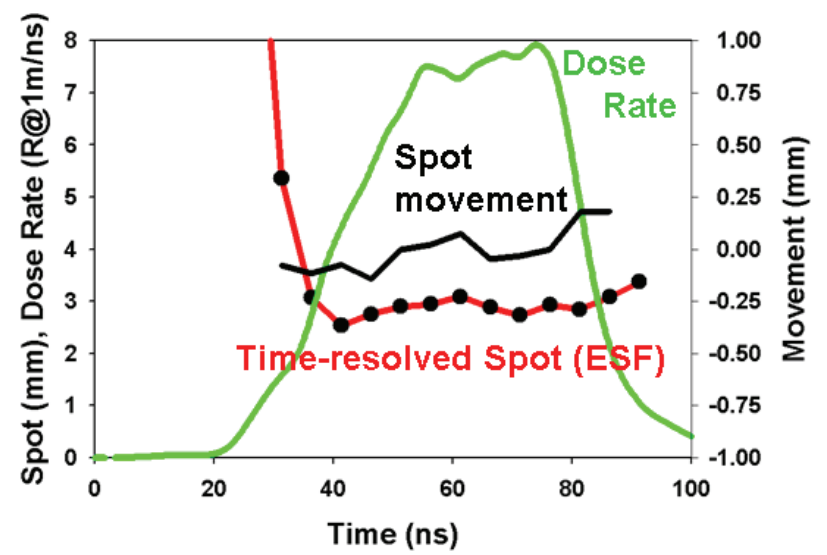

Figure 10. Measurement of time-resolved x-ray spot size, dose rate, and x-ray source movement using the TRSD.

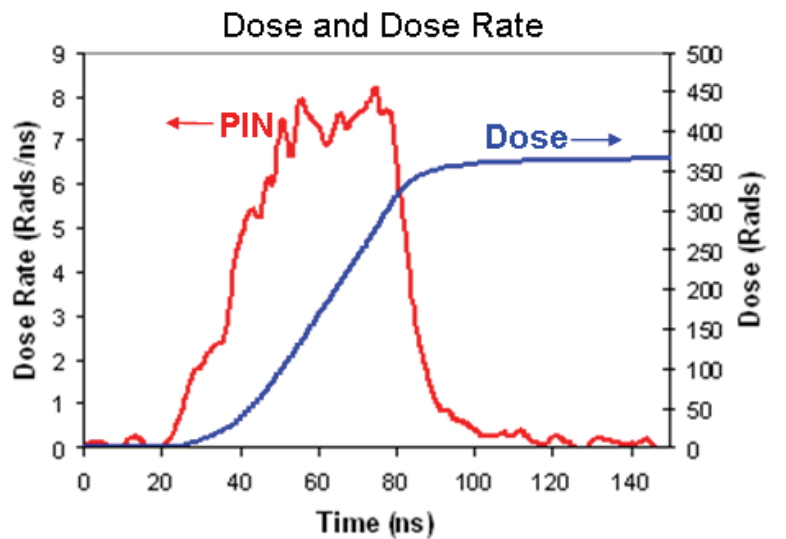

Figure 11. Dose rate (red) and dose (blue) from p-i-n diode and TLD measurements.

\section{IV.PLASMA DIAGNOSTICS}

Characterizations of the plasmas that evolve into the AK gap are performed using a combination of gated optical imaging and spectroscopy diagnostics [11]. Fastgated images of the visible plasma light taken during the early and middle times of the $\mathrm{x}$-ray pulse are shown in Fig. 12. Early in the pulse, light is observed at the cathode tip and a bright spot at the anode correlated with the x-ray source location. By the middle of the pulse at the time of peak radiation, the light from both regions has expanded and a bright channel extends across the entire AK gap with an estimated plasma density of at least $10^{16} \mathrm{~cm}^{-3}$.

Spectroscopy measurements are performed using a focused array of scintillator fibers and individual fibers both on and off-axis as shown in Fig. 13. Fast-gated and streak cameras record the spectra over the visible and very near UV wavelength ranges during and after the x-ray pulse. Key observations are as follows. Plasmas evolve from both the cathode and anode and are composed of surface contaminants $\left(\mathrm{CH}^{\prime} \mathrm{s}, \mathrm{H}_{2} \mathrm{O}\right)$ and metals associated with the electrodes ( $\mathrm{Al})$. During the $\mathrm{x}$-ray pulse, strong continuum emission is observed on-axis. After the x-ray pulse ( $100 \mathrm{~ns}$ ) and off-axis (few mm's), increased continuum emission is observed as well as distinct line emission from Al II and III and C III and IV charge states. Using this late-time spectra, calculations of the plasma densities and temperatures are performed to effectively calibrate the continuum intensity which is then correlated to the continuum measured during the $\mathrm{x}$-ray pulse. The estimated plasma density within a few mm's of the anode during the $\mathrm{x}$-ray pulse is up to $\sim 10^{17} \mathrm{~cm}^{-3}$. The calculations assume a 5-mm diameter plasma channel onaxis based on the optical images. Because of the low percentages of ion constituents measured after the pulse, the plasma during the pulse appears to be primarily ( $>95$ $\%)$ protonic. 

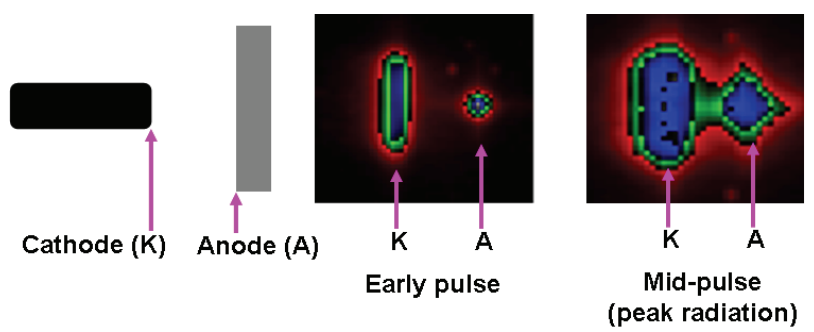

Figure 12. Gated optical images of the visible plasma light taken during the $\mathrm{x}$-ray pulse.

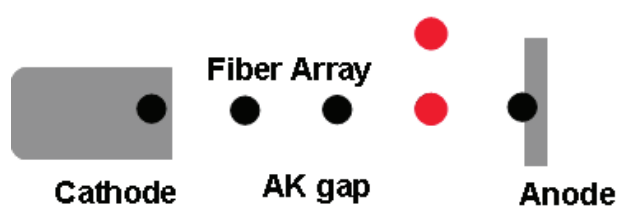

Figure 13. Layout of focused scintillator fibers for spectroscopy measurements.

\section{LSP SIMULATIONS OF SMP DIODE}

Hybrid PIC/fluid simulations using the LSP code were performed to model the SMP diode in the presence of an anode plasma. The simulations are performed in a 2-d $(\mathrm{r}, \mathrm{z})$ axis-symmetric geometry. For the simulations, a 20ns linear rise to 7-MV flat top voltage was applied. The plasma creation is modeled via electron beam bombardment of the anode surface which is legislated with 20 monolayers of e-H+ desorbed over $30 \mathrm{~ns}$.

The simulations reproduce relevant diode operating parameters from the experiments including the current and impedance behavior. The electron beam strikes the anode and quickly heats the surface resulting in ion emission and plasma evolution. The calculated ion currents are typically $15 \%$ of the total diode current. Particle density plots of the cathode electrons and anode protons are shown in Fig. 14. The electron beam focal position remains fixed on the anode target over the 60 -ns simulation time, which compares well with the timeresolved spot measurements. The simulations suggest that the anode plasma expands as an annulus with densities in the mid- $10^{15} \mathrm{~cm}^{-3}$ range extending into the AK gap at large radius. In addition, ions emitted from the dense plasma tend to coalesce on-axis forming a smalldiameter $(\sim 0.1 \mathrm{~mm})$, high-density $\left(\sim 10^{17} \mathrm{~cm}^{-3}\right)$ plasma channel with $\sim \mathrm{MeV}$ directed energy.

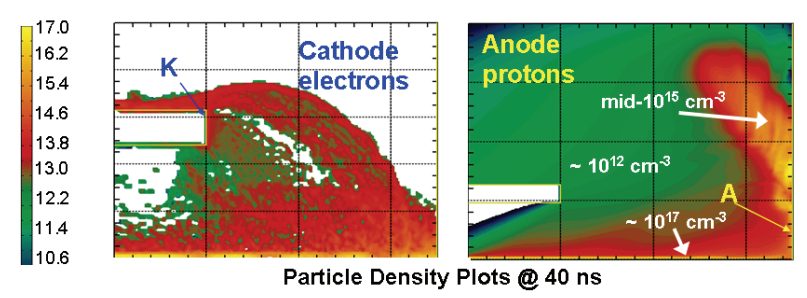

Figure 14. Particle density plots from LSP simulation at $40 \mathrm{~ns}$ of cathode electrons and anode protons.

\section{VI.SUMMARY}

SMP diode experiments have been performed on the RITS-6 accelerator. Using the 40-ohm MITL configuration, the diode operates at voltages between 6-7 MV as inferred from simulations and radiographer's equations. The diode yields over350 R@1m in a sub 3$\mathrm{mm}$ x-ray spot. Electrode plasmas evolve into the AK gap during the pulse as indicated by gated optical images and spectroscopic measurements. The spectroscopic measurements reveal that high-density plasmas in the range $\sim 10^{16} \mathrm{~cm}^{-3}$ extend across the AK gap by the middle of the pulse and also show that high densities $\sim 10^{17} \mathrm{~cm}^{-3}$ exist with a few mm's of the anode surface during the pulse. Simulations of the anode plasmas using the LSP code suggest a more complicated plasma evolution comprised of a moderately dense $\sim$ mid- $10^{15} \mathrm{~cm}^{-3}$ annular plasma evolving from the anode as well as a very narrow $\sim 0.1$-mm, high-density $\sim 10^{17} \mathrm{~cm}^{-3}$ plasma jet on-axis with high-directed energy $>>\mathrm{keV}$. Future work will focus on obtaining better measurements of the plasma parameters during the pulse and comparing to simulations. Future work is also being directed towards characterizing the spectral output of the $\mathrm{x}$-ray source in order to correlate with the diode operation.

\section{REFERENCES}

[1] D. Johnson, et al. in Proc. of the 15th IEEE Int. Pulsed Power Conf. (IEEE, 2005), pp. 314-317.

[2] LSP is a software product developed by ATK Mission Research, Albuquerque, NM 87110.

[3] J. M. Creedon, Appl. Phys. 48, 1070 (1977).

[4] C. W. Mendel and S. E. Rosenthal, Phys. Plasmas 2, 1332 (1995).

[5] I. Crotch, private communication, AWE.

[6] J.F. Briesmeister, Ed., LA-13709-M, Los Alamos National Laboratory (March, 2000).

[7] D. Hinshelwood, et al., IEEE Trans. Plasma Sci., 35, 565 (2007).

[8] J. A. Halbleib and W. H. Vandevender, Nucl. Sci. and Eng. 61, 288 (1976).

[9] S. Portillo, et al., IEEE Trans. Plasma Sci., 34, 1908 (2006).

[10] T. Goldsack, et al., IEEE Trans. Plasma Sci., 30, 239 (2002).

[11]M. D. Johnston, et al., 35th IEEE International Conference on Plasma Science (ICOPS, Karlsruhe, Germany June 2008). 